on the roles were also investigated, with in some cases two experimenters giving conflicting orders, in others the experimenter himself offering to be the 'learner' to show how harmless everything was and then screaming to be let out, and so on. The results seem clear: given a defined chain of command and a guiding plan which must be obeyed most subjects were prepared to administer severe pain in a futile 'experiment' even when it caused them considerable emotional distress. In the most extreme cases, people were found who pressed on even when they thought the 'victim' had a weak heart and might be dying.

Thus far, the book is compelling reading and provides food not just for thought but for self analysis, but when Professor Milgram feels the need to produce theories to explain the behaviour he is on much weaker ground. This is as much because of the state of the art of psychology as through any fault of his, but it is a pity that able experimenters should so often feel the need to be theoreticians as well. The latter part of the book is, however, prevented from being in any sense an anticlimax by a double sting in the tail--an epilogue about My Lai, and an appendix discussing the ethics of carrying out the experiment at all. MARY GRIBBIN

\section{Eyes of the aged}

The Human Lens in Relation to Cataract. Pp. $\mathrm{x}+324$. (Ciba Foundation Symposium 19, New Series.) (Elsevier/ Excerpta Medica/North-Holland, Associated Scientific Publishers: Amsterdam, London and New York, 1973.) Dfl. $43.50 ; \$ 16.70$.

Although the surgery of cataracts figures prominently in many ophthalmic practices, clinical research has been mainly concerned with the technological problems of avoiding the post-operative complications. The relative success of modern cataract surgery may have inhibited to some extent the need for research into the mechanisms responsible for, and the changes which occur as a result of, the development of lens opacities in the aged. There cannot be complete complacency on the clinical side, however, as the patient after a successful lens extraction is nevertheless challenged by a series of problems in achieving 'better sight'. The need for research at a more fundamental level is thus of great importance.

Publication of this volume was timed to coincide with the retirement of Dr A. Pirie from the post of Reader to the University of Oxford, which she has held with distinction from 1948 to 1973 , and records the proceedings of a symposium on the human lens in relation to cataract under her chairmanship. In a series of papers followed by wide ranging discussions investigators eminent in their respective fields present their current views arising from investigations into the physical, biochemical, immunological and epidemiological changes which occur in the ageing lens. In subsequent sections it is perhaps unfortunate that the problem of iatrogenic cataracts did not receive more attention, but this was more than offset by a communication indicating that at least in certain parts of the world the age at which cataracts develop in populations depends on the geographical habitat.

This volume will be of value to cataract surgeons who wish to expand their knowledge of the subject beyond the confines of operative technique, and provides an up-to-date review of concepts and investigation techniques for laboratory workers engaged in research on lenticular opacities in the aged.

$$
\text { J. C. DeAn HarT }
$$

\section{Ions enter crystals}

Channeling: Theory, Observation and Applications. Edited by D. V. Morgan. Pp. xii +905 . (Wiley: London and New York, November 1973.) £11.75.

THE discovery in 1965 that under certain conditions fast ions from nuclear particle accelerators could penetrate to anomalously great depths in single crystals came as something of a surprise to physicists. It was not that any new principle was involved but simply that the passage of fast ions through solids had been well studied for some 70 years and it was not to be expected that a phenomenon as striking as channelling could have avoided detection for so long. This greatly increased penetration occurs when the charged particles travel along the directions of the strings of atoms which make up the crystal lattice, for in these directions the particles can travel freely in the open channels between the atoms with little energy loss. In the past eight years there has been a considerable amount of research on the channelling effect and the principal features are now well understood. Since the effect is beginning to find a number of applications the appearance of a comprehensive treatment is both welcome and timely.

The first half of the book is devoted to discussing the fundamental aspects of channelling and of the corresponding blocking phenomenon which gives rise to the distinctive pattern of ions backscattered by single crystals. Classical models provide a good account of these processes and computer simulation has been successfully employed for predicting the trajectories of ions in crystals. With increasing accuracy of measurement, however, the use of a wave description may become necessary as in the case of channelling of electrons.

The second half of the book contains accounts of a number of the applications in which the phenomenon has been used. Thus since the blocking process depends sensitively on the structure of the solid it can be used for crystallographic analysis, the pattern immediately leading to the identification of the principal planes and axes of the crystal. Again impurities embedded in otherwise perfect crystals obstruct the free passage of the channelled ions and the nature and location of these impurities can be determined by the change in the pattern of the scattered ions. This technique of atom location is already finding many uses and includes the fields of radiation damage, surface physics and semiconductor technology, each of which is discussed at some length.

One historically interesting application lies in the field of nuclear physics and is for measuring time intervals as short as $10^{-18} \mathrm{~s}$; this was devised in 1960 by Dr P. B. Treacy, five years before the phenomenon of channelling had been discovered. The principle of the method was to observe the distance travelled by an excited nucleus in a crystal in the interval between excitation and disintegration. Dr Treacy's assumption was that the decay products would have a greater chance of escaping from a crystal if the nucleus disintegrated at a point in a channel rather than at the lattice point where it was excited. His experiment proved abortive owing presumably to the poor quality of his crystals and no effect was seen. Some years later, however, when the channelling phenomenon was known, this application was independently proposed by other authors and shown to work. It has now become an established technique and some beautiful experiments have been carried out using it.

The whole field of channelling is excellently described in this book. Its multiple authorship ensures that the chapters on the different aspects of the field bear the stamp of authority and yet they are sufficiently linked together to make a coherent whole without destroying the pleasant idiosyncrasies in style of the individual authors; the editor is to be congratulated on his successful blending together of the component parts. It is informative, stimulating and well presented. As a library book it is likely to remain of value for some time and should be available to all. It will be of special interest to those working in the fields of solid state physics and of the science of materials. 